\title{
GeV Gamma Rays from Supernova Remnants Interacting with Molecular Clouds
}

\author{
Yasunobu Uchiyama*t \\ SLAC National Accelerator Laboratory, Stanford University \\ E-mail: uchiyamaeslac.stanford.edu
}

\section{On behalf of the Fermi-LAT Collaboration}

\begin{abstract}
We report the current status of the observations of supernova remnants (SNRs) with the Large Area Telescope (LAT) aboard the Fermi Gamma-ray Space Telescope, focusing on middle-aged SNRs that appear to be interacting with molecular clouds. Observations with the Fermi LAT in an energy range from $0.2 \mathrm{GeV}$ to $\sim 100 \mathrm{GeV}$ have unveiled the presence of luminous $\mathrm{GeV}$ gamma-ray emission in middle-aged SNRs, providing a new insight into the shock-acceleration theory and the origin of galactic cosmic rays. The middle-aged SNRs detected by the Fermi LAT are generally much brighter in $\mathrm{GeV}$ than in $\mathrm{TeV}$ in terms of energy flux, which emphasizes the importance of the $\mathrm{GeV}$ observations. Spectral analysis of the bright objects revealed spectral steepening in the Fermi-LAT band. Remarkably, most (if not all) of them are known to be interacting with molecular clouds, and they are also the strong sources of synchrotron radio emission. We discuss possible scenarios to explain the enhanced $\mathrm{GeV}$ gamma-ray emission in the cloud-interacting SNRs. Particular emphasis is placed on a scenario in which shock-accelerated cosmic-rays are adiabatically compressed and energized as result of radiative cooling behind the cloud shock.
\end{abstract}

25th Texas Symposium on Relativistic Astrophysics

December 6-10, 2010

Heidelberg, Germany

* Speaker.

†Panofsky Fellow. 


\section{Introduction}

Diffuse shock acceleration [1] operating at a collisionless shock produces nonthermal particles in a supernova remnant (SNR). Remarkable progress in the observations of shock-accelerated particles was made in the last decade, especially in the X-ray and $\mathrm{TeV} \gamma$-ray bands, which has strengthened the conjecture that shock acceleration in SNRs is responsible for the bulk of the galactic cosmic rays (GCRs). Synchrotron X-ray emission produced by multi-TeV electrons has been detected from most of young historical SNRs [2]. The X-ray measurements by the Chandra X-ray Observatory with its arcsecond resolution imaging have shown that particle acceleration to $\mathrm{TeV}$ energies at strong shocks in young SNRs accompanies significant amplification of interstellar magnetic fields [3]. Direct evidence for the production of multi-TeV particles in shell-type SNRs came from the $\mathrm{TeV} \gamma$-ray observations made with ground-based Cherenkov telescopes such as H.E.S.S. [4], though the emission mechanism ("hadronic vs leptonic") remains unsettled. Gamma-ray observations at the $\mathrm{GeV}$ band have been expected to provide complementary information on particle acceleration at the expanding shock waves of galactic SNRs.

In the $\mathrm{GeV} \gamma$-ray band, possible associations of $\gamma$-ray sources with five radio-bright shell-type SNRs were reported by Ref. [5] based on the data taken in 1991-1994 by the EGRET instrument onboard the Compton Gamma Ray Observatory (see also Ref. [6). The two strongest $\gamma$-ray sources that are possibly associated with SNRs are the EGRET unidentified sources in the directions of $\gamma$ Cygni and IC 443, both of which are known to be interacting with adjacent molecular clouds. If the $\gamma$-ray excesses are due to cosmic-ray interactions with a dense cloud, the dominant emission processes are either bremsstrahlung by relativistic electrons or $\pi^{0}$-decay $\gamma$-rays resulting from proton-proton hadronic collisions. However, the EGRET point-spread function is much wider than the spatial extent of most of the SNRs with possible $\gamma$-ray associations, which made it difficult to preclude the possibility that the $\gamma$-ray sources are due to pulsars ${ }^{1}$.

The Large Area Telescope (LAT) onboard the Fermi Gamma-ray Space Telescope [8], which was launched in June 2008, has started to explore the GeV $\gamma$-ray sky with much improved resolving power and unprecedented sensitivity. The observations with the Fermi-LAT provide new methods of addressing unsolved problems in shock acceleration theory and the origin of the GCRs. Using the LAT data from the first $\sim 1$ yr of observations, the Fermi LAT Collaboration has reported the detections of $\gamma$-ray emission from several SNRs [9-14]. Middle-aged SNRs interacting with molecular clouds (MCs) constitute the dominant class of $\gamma$-ray-luminous SNRs. Here we summarize the results for such MC-interacting SNRs obtained with the LAT and discuss the mechanisms by which $\gamma$-ray emission can be enhanced in such systems.

\section{Fermi-LAT Detections of SNRs Interacting with Molecular Clouds}

The Fermi LAT Collaboration has so far reported the discoveries of spatially extended $\gamma$-ray sources coincident with four remnants: W51C [9], W44 [11], IC 443 [12], and W28 [13]. They are middle-aged $\left(\sim 10^{4} \mathrm{yr}\right)$ remnants that appear to be interacting with molecular clouds. Figure 1 shows a collection of the $2-10 \mathrm{GeV} \gamma$-ray count maps obtained with $2.5 \mathrm{yr}$ data of the LAT. For

\footnotetext{
${ }^{1}$ One of the two most significant EGRET sources (a source in the $\gamma$ Cygni SNR) is indeed found to be a $\gamma$-ray pulsar with the Fermi LAT [7.
} 


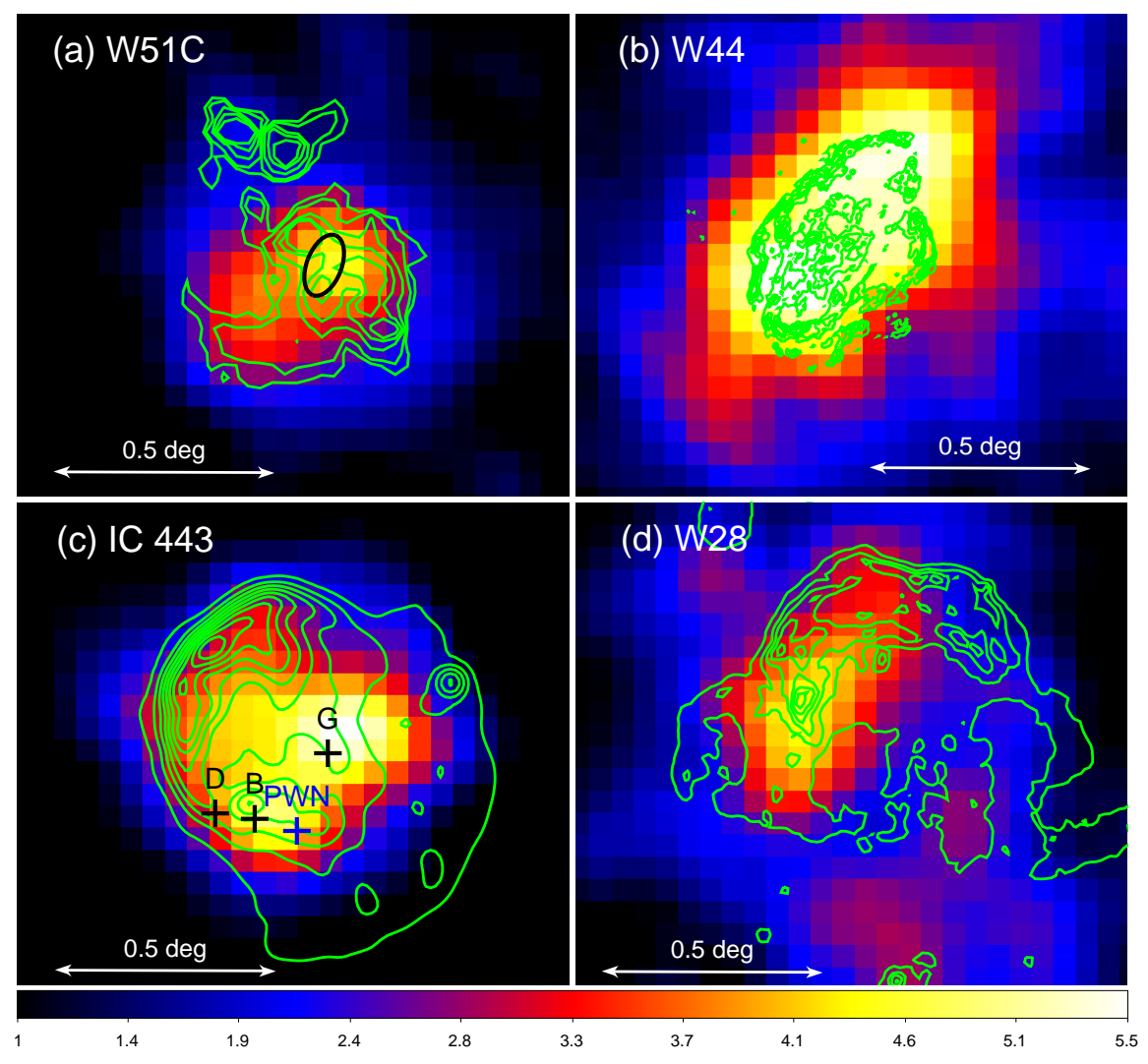

Figure 1: Fermi LAT count maps in $2-10 \mathrm{GeV}$ of the MC-interacting SNRs with extended $\gamma$-ray emission: (a) W51C; (b) W44; (c) IC443; and (d) W28. The LAT count maps (in the celestial coordinates) constructed from front-converted events in the four panels are smoothed by a Gaussian kernel of $0.15 \mathrm{deg}$. The intensity is in units of counts per pixel with a pixel size of $0.05 \times 0.05 \mathrm{deg}^{2}$, and shown in a common range of 1.0-5.5 counts per pixel. Superposed are the contours from the VLA radio maps (see the text). A black ellipse in panel (a) represents the location of shocked CO clumps [15]. The black crosses in panel (c) are the locations of shocked molecular clumps from which $\mathrm{OH}$ maser is detected [16]. The position of a pulsar wind nebula is also marked in panel (c).

better spatial resolution, only front-converted events are used. Even without subtracting the intense Galactic diffuse emission, significant $\gamma$-ray emission clearly stands out in excess of the background diffuse emission. In each panel, superimposed is a radio continuum map synthesized with the Very Large Array (VLA) at a frequency of: (a) $1.4 \mathrm{GHz}$ [17]; (b) $1.4 \mathrm{GHz}$ (The FIRST Survey); (c) $330 \mathrm{MHz}$ [16]; and (d) $330 \mathrm{MHz}$. The spatial extent of the $\gamma$-ray emission estimated through the maximum likelihood technique was generally found to be consistent with the size of the radio remnant. Given the size of a point-spread function of the LAT in this energy band, dedicated investigations are necessary to establish possible sub-structures within these remnants, which will be reported elsewhere.

The radio and $\gamma$-ray properties of the four SNRs are similar in many aspects. The synchrotron radio emission has a large flux of 160-310 Jy at $1 \mathrm{GHz}$ with flat spectral index of $\alpha \simeq 0.3-0.4$ 


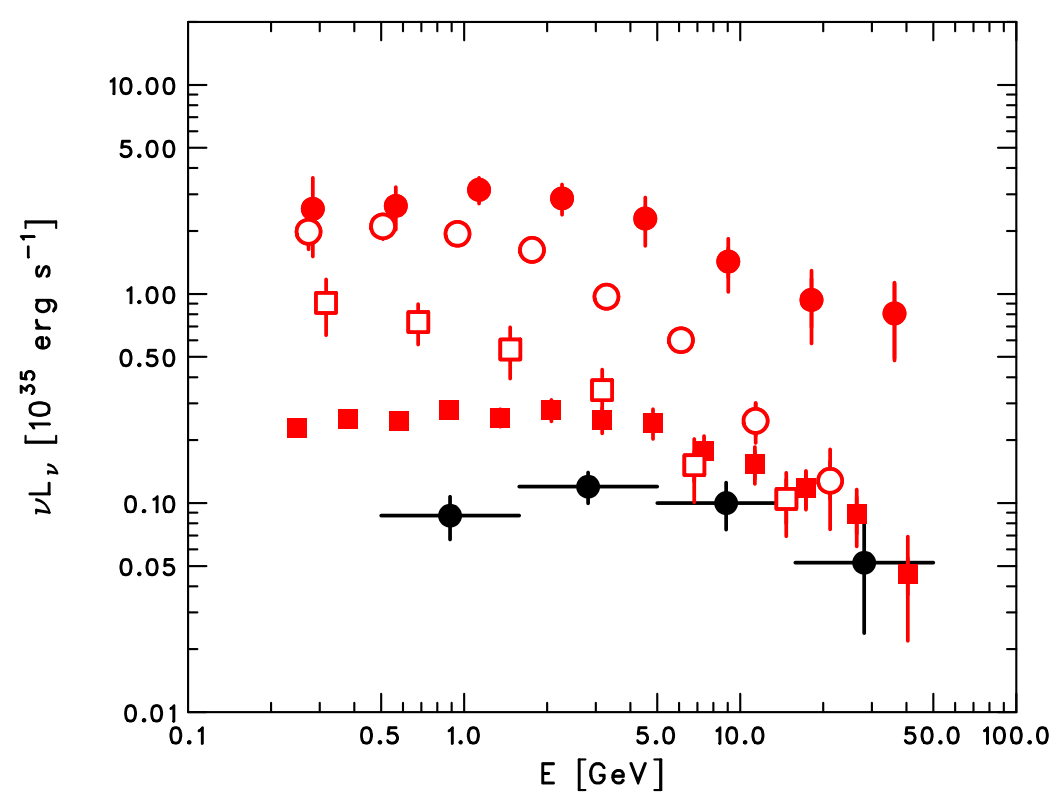

Figure 2: Gamma-ray spectra of shell-type SNRs measured with the Fermi LAT: W51C (red filled circles [9]); W44 (red open circles [11]); IC 443 (red filled rectangles [12]); W28 (red open rectangles [13]); Cassiopeia A (black filled circles [10).

[18]. The GeV $\gamma$-ray spectrum commonly exhibits a spectral break at around $1-10 \mathrm{GeV}$, and the luminosity ranges $L_{\gamma}=10^{35-36} \mathrm{erg} \mathrm{s}^{-1}$. In Fig. 2 the $\gamma$-ray spectra of the MC-interacting SNRs measured with the LAT are shown in the so-called $v L_{v}$ form along with the spectrum of Cas A. The distances are taken from the nominal values described in Refs. [9- 13]. Note that the $\gamma$ ray luminosities of W51C and W44 are more than an order of magnitude larger than the $\gamma$-ray luminosity of Cas A.

The LAT-detected SNRs are generally radio-bright objects, which suggests some physical link between the synchrotron radiation and the $\mathrm{GeV} \gamma$-rays. Indeed, the median value of the radio surface brightness $^{2}$ at $1 \mathrm{GHz}$ of the Galactic SNRs is $\Sigma \simeq 2 \times 10^{-9} \mathrm{erg} \mathrm{cm}^{-2} \mathrm{~s}^{-1} \mathrm{sr}^{-1}$ [18], which is an order of magnitude smaller than that of the LAT-detected SNRs. Moreover, there found a possible correlation between the radio and $\gamma$-ray fluxes from MC-SNRs. Figure 3 (left) shows a plot of the radio flux vs $\gamma$-ray flux for the current list of MC-SNRs that are detected by the LAT [9-14, 19. The radio and $\gamma$-ray energy fluxes represent the total fluxes integrated over a remnant. In Fig. 3 (right), a comparison between the radio and $\gamma$-ray intensities is made in terms of mean surface brightness, where the total flux is divided by the solid angle subtended by each remnant. The mean surface brightness, which is distance-independent, has a large dynamic range over more than two orders of magnitude.

\footnotetext{
${ }^{2}$ Usually, the radio surface brightness is represented as $\Sigma_{v} \equiv f_{V} / \Omega$ in units of $\mathrm{W} \mathrm{m}^{-2} \mathrm{~Hz}^{-1} \mathrm{sr}^{-1}$. Here we adopt $\Sigma \equiv v \Sigma_{v}=v f_{v} / \Omega$ in units of erg $\mathrm{cm}^{-2} \mathrm{~s}^{-1} \mathrm{sr}^{-1}$.
} 

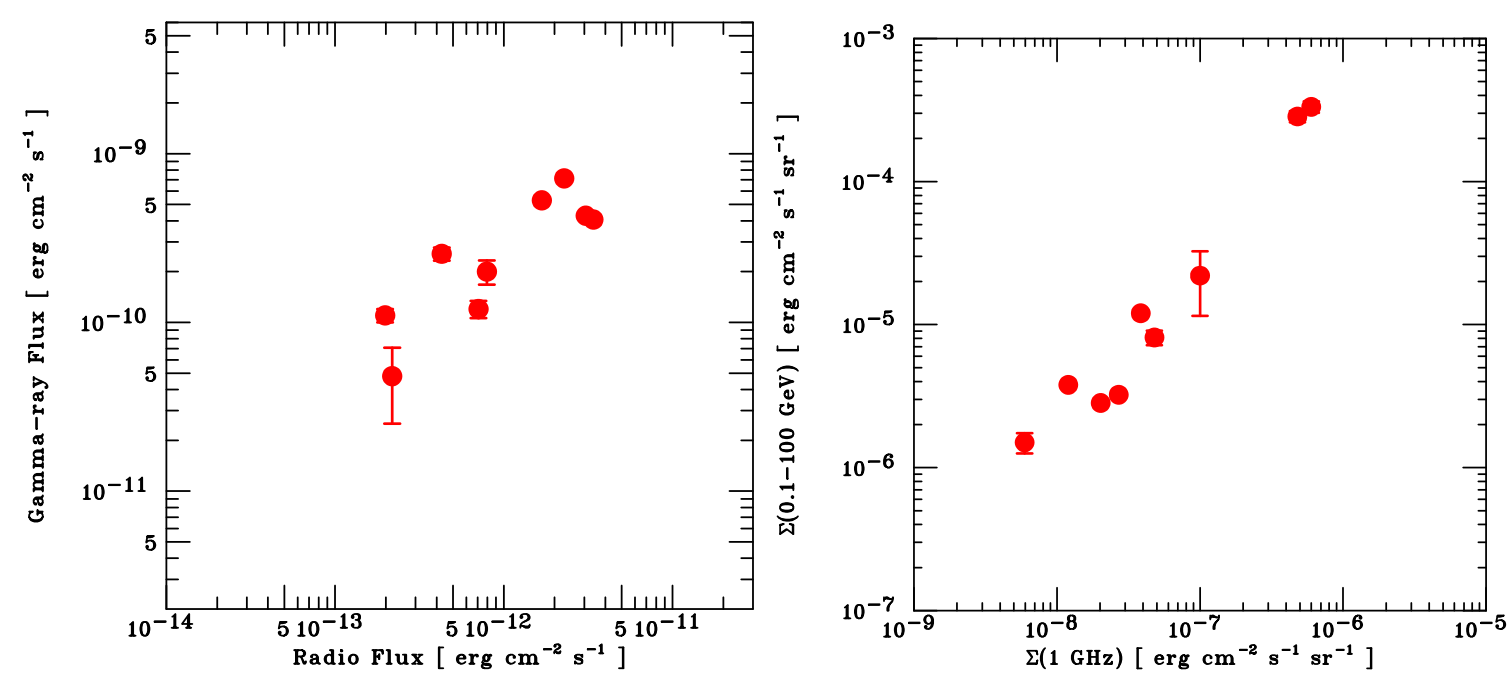

Figure 3: (Left) Radio flux (synchrotron) vs $\mathrm{GeV} \gamma$-ray flux for MC-interacting SNRs. The $\gamma$-ray energy flux integrated over $0.1-100 \mathrm{GeV}$ and the radio flux, $v f_{v}$ at $1 \mathrm{GHz}$, are shown. (Right) Mean surface brightness of the synchrotron radio emission and $\mathrm{GeV} \gamma$-ray emission. The flux-flux plot is converted into this form using the solid angles of the radio remnants.

\section{Discussion}

Since the interaction with a molecular cloud clearly plays a key role in enhancing the $\gamma$-ray emission, bremsstrahlung by relativistic electrons or $\pi^{0}$-decay $\gamma$-rays produced by high-energy protons are the two plausible dominant channels of the $\gamma$-radiation. The observed large luminosity of the GeV $\gamma$-ray emission precludes the inverse-Compton scattering off the CMB and interstellar radiation fields as the main emission mechanism. For instance, the luminosity of SNR W51C requires an unacceptably large amount of the total CR electron energy of $W_{e} \simeq 1 \times 10^{51} \mathrm{ergs}$ [9]. Generally the high-energy protons are thought to be the predominant component of accelerated particles in SNRs so that the $\pi^{0}$-decay $\gamma$-ray emission is stronger than the electron bremsstrahlung (see however Ref. [20]).

There are two different types of scenarios to explain the $\mathrm{GeV} \gamma$-ray emission arising from the MC-SNR systems. The Runaway CR model considers $\gamma$-ray emission from molecular clouds illuminated by runaway CRs that have escaped from their accelerators [21-23]. CR particles released by a remnant diffuse into interstellar space with an energy-dependent diffusion coefficient, and collide with massive clouds producing $\gamma$-ray photons via $\pi^{0}$-decay (by CR hadrons) and bremsstrahlung (by CR electrons) channels. The $\gamma$-ray wide-band spectra from the $\mathrm{GeV}$ to $\mathrm{TeV}$ energies can markedly vary from one object to another depending on the relative locations of the accelerator and the target cloud as well as the time history of the accelerator. For example, a hard $\mathrm{GeV} \gamma$-ray source [13] which is also bright in TeV [24] outside the southern boundary of SNR W28 may be explained by runaway CRs. Future analysis of spatial distributions of GeV $\gamma$-rays combined with $\mathrm{CO}$ observations would make it possible to discern the $\gamma$-ray sources that are attributable to runaway CRs. Then one can learn about how CRs are released into interstellar space and how they propagate in the vicinity of the SNR where self-generated Alfvén waves may change the local diffusion coefficient [25]. 
Another scenario, the Crushed Cloud model [26] invokes a "shocked" molecular cloud into which a radiative shock (typically $v_{\mathrm{s}} \sim 100 \mathrm{~km} \mathrm{~s}^{-1}$ ) is driven by the high pressure of hot plasmas in the blastwave region. The cosmic-ray particles accelerated at a cloud shock are adiabatically compressed behind the shock front, resulting in enhanced synchrotron and $\pi^{0}$-decay $\gamma$-ray emissions [27]. It was shown by Ref. [26] that reacceleration of pre-existing CR electrons and protons is capable of explaining both radio and $\mathrm{GeV} \gamma$-ray spectra using reasonable sets of parameters. The $\gamma$-ray luminosity of $L_{\gamma} \sim 10^{35} \mathrm{erg} \mathrm{s}^{-1}$ in $1-10 \mathrm{GeV}$ agrees well with the theoretical expectation. In this scenario, the radio and $\gamma$-ray emissions from the MC-SNRs are intimately connected, and therefore the possible radio-gamma correlation can be readily explained.

The Runaway CR and Crushed Cloud models are not mutually exclusive. The former presumes unshocked molecular clouds as the $\gamma$-ray production sites while the latter considers shocked clouds. Both unshocked and shocked clouds could be the important sites of the $\gamma$-ray production. Also, in addition to the pre-existing GCRs in a molecular cloud, runaway CRs released from a shock may make a large contribution to the cosmic-ray density in the molecular cloud particularly at $\mathrm{TeV}$ energies. When such a cloud is struck and compressed by the blastwave, the $\gamma$-ray flux at high energies should be enhanced with respect to what is presented in the model of Ref. [26].

\section{Acknowledgments}

The Fermi LAT Collaboration acknowledges support from a number of agencies and institutes for both development and the operation of the LAT as well as scientific data analysis. These include NASA and DOE in the United States, CEA/Irfu and IN2P3/CNRS in France, ASI and INFN in Italy, MEXT, KEK, and JAXA in Japan, and the K. A. Wallenberg Foundation, the Swedish Research Council and the National Space Board in Sweden. Additional support from INAF in Italy and CNES in France for science analysis during the operations phase is also gratefully acknowledged.

\section{References}

[1] Blandford, R., \& Eichler, D., Particle acceleration at astrophysical shocks: A theory of cosmic ray origin, Physics Reports, 154, 1-75, 1987.

[2] Reynolds, S.P., Supernova Remnants at High Energy, ARAA, 46, 89-126, 2008.

[3] Uchiyama, Y., Aharonian, F.A., Tanaka, T., et al., Extremely fast acceleration of cosmic rays in a supernova remnant, Nature, 449, 576-578, 2007.

[4] Aharonian, F.A., Akhperjanian, A.G., Aye, K.M., et al., High-energy particle acceleration in the shell of a supernova remnant, Nature, 432, 75-77, 2004.

[5] Esposito, J.A., Hunter, S.D., Kanbach, G., Sreekumar, P., EGRET Observations of Radio-Bright Supernova Remnants, ApJ, 461, 820-827, 1996.

[6] Torres, D.F., Romero, G.E., Dame, T.M., et al., Supernova remnants and gamma-ray sources, Phys. Rep., 382, 303-380, 2003.

[7] Abdo, A.A., Ackermann, M., Ajello, M., et al., The First Fermi Large Area Telescope Catalog of Gamma-ray Pulsars, ApJS, 187, 460-494, 2010.

[8] Atwood, W.B., Abdo, A.A., Ackermann, M., et al., The Large Area Telescope on the Fermi Gamma-Ray Space Telescope Mission, ApJ, 697, 1071-1102, 2009. 
[9] Abdo, A.A., Ackermann, M., Ajello, M., et al., Fermi LAT Discovery of Extended Gamma-Ray Emission in the Direction of Supernova Remnant W51C, ApJL, 706, 1-6, 2009.

[10] Abdo, A.A., Ackermann, M., Ajello, M., et al., Fermi-LAT Discovery of GeV Gamma-Ray Emission from the Young Supernova Remnant Cassiopeia A, ApJL, 710, 92-97, 2010.

[11] Abdo, A.A., Ackermann, M., Ajello, M., et al., Gamma-Ray Emission from the Shell of Supernova Remnant W44 Revealed by the Fermi LAT, Science, 327, 1103-1106, 2010.

[12] Abdo, A.A., Ackermann, M., Ajello, M., et al., Observation of Supernova Remnant IC 443 with the Fermi Large Area Telescope, ApJ, 712, 459-468, 2010.

[13] Abdo, A.A., Ackermann, M., Ajello, M., et al., Fermi Large Area Telescope Observations of the Supernova Remnant W28 (G6.4-0.1), ApJ, 718, 348-356, 2010.

[14] Abdo, A.A., Ackermann, M., Ajello, M., et al., Fermi-LAT Study of Gamma-ray Emission in the Direction of Supernova Remnant W49B, ApJ, 722, 1303-1311, 2010.

[15] Koo, B.-C., \& Moon, D.-S. Interaction between the W51C Supernova Remnant and a Molecular Cloud. II. Discovery of Shocked CO and HCO+, ApJ, 485, 263-269, 1997.

[16] Hewitt, J.W., Yusef-Zadeh, F., Wardle, M., et al., Green Bank Telescope Observations of IC 443: The Nature of $\mathrm{OH}(1720 \mathrm{MHz})$ Masers and OH Absorption, ApJ, 652, 1288-1296, 2006.

[17] Koo, B.-C., \& Moon, D.-S., Interaction between the W51C Supernova Remnant and a Molecular Cloud. I. HI 21 Centimeter Line Observations, ApJ, 475, 194-210, 1997.

[18] Green, D.A., A revised Galactic supernova remnant catalogue, Bulletin of the Astronomical Society of India, 37, 45-61, 2009.

[19] Castro, D., \& Slane, P., Fermi Large Area Telescope Observations of Supernova Remnants Interacting with Molecular Clouds, ApJ, 717, 372-378, 2010.

[20] Bykov, A.M., Chevalier, R.A., Ellison, D.C., \& Uvarov, Y.A., Nonthermal Emission from a Supernova Remnant in a Molecular Cloud, ApJ, 538, 203-216, 2000.

[21] Aharonian, F.A., \& Atoyan, A.M., On the emissivity of $\pi^{0}$-decay gamma radiation in the vicinity of accelerators of galactic cosmic rays, A\&A, 309, 917-928, 1996.

[22] Gabici, S., Aharonian, F.A., \& Casanova, S., Broad-band non-thermal emission from molecular clouds illuminated by cosmic rays from nearby supernova remnants, MNRAS, 396, 1629-1639, 2009.

[23] Ohira, Y., Murase, K., \& Yamazaki, R., Gamma-rays from molecular clouds illuminated by cosmic rays escaping from interacting supernova remnants, MNRAS, 410, 1577-1582, 2011.

[24] Aharonian, F., Akhperjanian, A.G., Bazer-Bachi, A.R., et al., Discovery of very high energy gamma-ray emission coincident with molecular clouds in the W28 (G6.4-0.1) field, A\&A, 481, 401-410, 2008.

[25] Fujita, Y., Ohira, Y., \& Takahara, F., Slow Diffusion of Cosmic Rays Around a Supernova Remnant, ApJL, 712, 153-156, 2010.

[26] Uchiyama, Y., Blandford, R., Funk, S., Tajima, H., \& Tanaka, T., Gamma-ray Emission from Crushed Clouds in Supernova Remnants, ApJL, 723, 122-126, 2010.

[27] Blandford, R., \& Cowie, L., Radio emission from supernova remnants in a cloudy interstellar medium, ApJ, 260, 625-634, 1982. 